\title{
Time-dependent rotational stability of dynamic planets with viscoelastic lithospheres
}

${ }_{3}$ K.M. Moore ${ }^{1, *}$, N.-H. Chan ${ }^{2}$, A. Daradich ${ }^{3}$, J.X. Mitrovica ${ }^{1}$

${ }^{1}$ Department of Earth $\&$ Planetary Sciences, Harvard University, 20 Oxford Street, Cambridge, MA, USA 02138

${ }^{2}$ Department of Earth and Planetary Sciences, McGill University, Montreal, PQ, Canada H3A 0E8

${ }^{3}$ Department of Earth Sciences, University of Ottawa, Ottawa, ON, Canada K1N 6N5

*Corresponding author. Email address: kimberly.moore@g.harvard.edu (K.M. Moore) 
2 K.M. Moore, N.-H. Chan, A. Daradich \& J.X. Mitrovica suggest, in contrast, that TPW on Mars can continue over time scales on the order of the relaxation time of the lithosphere after load emplacement; for sufficiently high lithospheric viscosities, this time scale may be comparable to the age of the planet.

Key words: Rotational dynamics, Interiors, Mars

\section{Introduction}

Modern theories describing the rotational stability of terrestrial planets emerged from the insights of Gold (1955). He argued that the rotation axis of all such planets is inherently unstable because any stabilization provided by the rotational bulge is transient; that is, the rotational bulge will ultimately reorient perfectly to any new position of the rotation axis and the system will thus lose all memory of any prior rotational state. In this case, any isolated mass load will eventually migrate, through true polar wander (TPW), to the equator, and, furthermore, a set of evolving loads can trigger relatively rapid and large $\left(\sim 90^{\circ}\right)$ amplitude TPW events (Goldreich and Toomre, 1969).

Willemann (1984) introduced an important caveat to Gold's arguments. He argued that TPW would introduce permanent elastic stresses in an otherwise unstressed planetary lithosphere, and this so-called remnant bulge would introduce a memory of prior rotational states. The net TPW driven by a mass load would, in this case, be governed by a balance between the centrifugal forces driving the mass load toward the equator and the stabilization of the rotation axis associated with the remnant bulge. Willemann's original derivation predicted that the remnant bulge stabilization was independent of the elastic thickness of the lithosphere. However, Matsuyama et al. (2006) corrected several minor errors in the derivation and his final expression for TPW revealed an important dependence on this thickness.

The above studies all considered the equilibrium position of the rotation axis; that is, the final position of the pole after all viscous stresses within the planet had fully relaxed. The speed of TPW will be a strong function of the mass of the evolving loads and the viscosity of the planetary interior, and various methods have been developed to incorporate this time history into a rotational stability theory in the absence of remnant bulge stabilization (e.g., Ricard et al., 1993; Tsai and Stevenson, 2007). Chan et al. (2011) adapted a linearized theory of TPW developed to study ice age Earth rotation (Sabadini and Peltier, 1981; Sabadini et al., 1982, Wu and Peltier, 1984; Vermeersen and Sabadini, 1999; Mitrovica et al., 2005) to consider the impact of remnant bulge stabilization on time-dependent polar wander. Their theory was valid for small displacements of the pole, and they demonstrated that the range 
of validity was a strong function of the location of the loads relative to the rotation axis. To overcome this small-angle limitation, Harada (2012), Creveling et al. (2012) and Chan et al. (2014) extended the pioneering approach of Ricard et al. (1993) to incorporate the effects of the remnant bulge on the predicted TPW history. In these studies, the lithosphere was treated as a purely elastic layer, with effectively infinite viscosity.

In reality, a lithosphere will have finite viscosity, and it will viscously relax in the case of a loading of sufficiently long time scale, and no rotational stability theory for terrestrial planets has yet considered this more realistic case. In this study, we extend the TPW theories of Ricard et al. (1993), Harada (2012), Creveling et al. (2012) and Chan et al. (2014) to include stabilization by a viscoelastic lithosphere of high (but finite) viscosity. On such a planet, TPW driven by loading of short time scale (i.e., a time scale shorter than the viscous relaxation time of the lithosphere) will be strongly stabilized by a remnant bulge, while on long time scales the lithosphere will provide no such stabilization and the physics described by Gold (1955) and Goldreich and Toomre (1969) will prevail. Our generalized theory accommodates both scenarios, and, more importantly, the stabilization associated with intermediate time scale loading.

In the next section we provide a short, schematic description of load-induced TPW on terrestrial planets in the case of no lithosphere, an elastic lithosphere, and a viscoelastic lithosphere. Following this, we derive the equations governing TPW in the last of these cases and consider the limiting cases of no lithosphere and an elastic lithosphere. We end with a section showing some numerical examples of Martian TPW that illustrate the theory.

\section{The Physics of TPW in the Presence of a Viscoelastic Lithosphere}

Gold (1955) considered TPW induced by the loading (i.e., mass redistribution) of a terrestrial planet possessing no lithosphere, as depicted in Figure 1A. To begin, we consider an initially non-rotating, spherical planet (A0). Next, the planet is spun up to some rotation rate and a rotational bulge is created that ultimately achieves an equilibrium form (A1). If a positive mass load is applied (A2), the rotation axis begins to adjust by moving away from this forcing (equivalently, in a reference frame fixed to the rotation axis, the load is thrown outwards toward the equator). This polar motion is initially resisted by the rotational bulge, but on a fluid planet the bulge will relax to match the new rotational state (A3). This process continues incrementally (A4) until, in the limit of long time, the load reaches the equator (A5). The rotation axis in the model of Gold is thus inherently unstable. While this model is only concerned with the final (equilibrium) form of the rotating body, Ricard et al. (1993) developed 


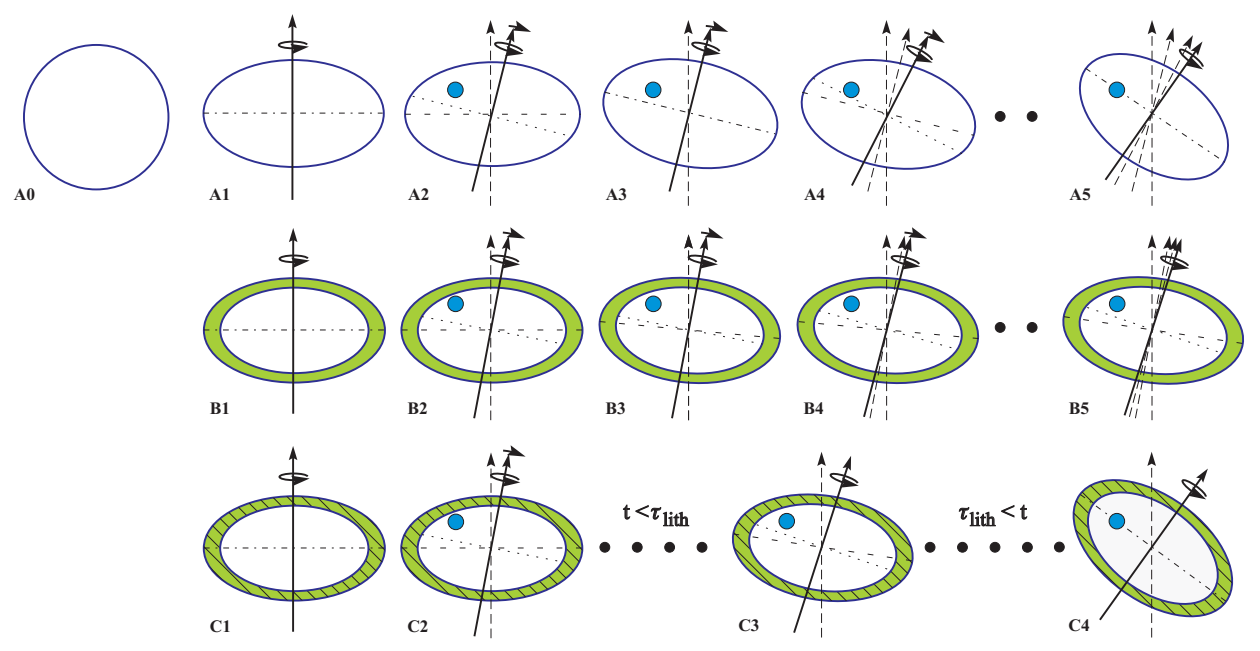

Figure 1. Schematic illustration of the physics of long-term TPW on terrestrial planets (A) without a lithosphere, (B) with an elastic lithosphere (represented by the solid green shell), and (C) with a viscoelastic lithosphere (green shell with hatching). All frames are drawn in a reference frame fixed to the load within the interior of the planet (blue dot). The solid arrow in each frame (other than A0) represents the contemporaneous rotation axis, while the dashed arrows symbolize previous locations of the rotation axis. The dotted line in the interior of the planet denotes the equator (i.e., the great circle $90^{\circ}$ from the rotation pole), while the dashed line is positioned to pass through the equator of the oblate form of the planet (i.e., it passes through the planet at its widest girth).

a non-linear rotational stability theory that mathematically captures the time dependence of the system associated with the evolution from (A1)-(A5). The theory assumes that the loading and response is slow enough that the rotation axis remains aligned with the principle axis of inertia.

Next, we consider TPW in the presence of an elastic (effectively infinite viscosity) lithosphere, the case treated by Willemann (1984). We begin with Figure 1A1, but assume that the planet develops an unstressed elastic lithosphere through slow cooling (B1). When a load is applied (B2), the rotation axis adjusts as before (the load is thrown toward the equator), but the polar motion is now resisted by elastic stresses induced in the lithosphere by the perturbed orientation of the centrifugal potential, i.e, the net TPW from the initial state is greater in A2 than B2. Willemann (1984) called this resistance the 'remnant bulge', since it will exist at any time in which the rotational bulge (or rotation axis) departs from its initial orientation. This process of gradual adjustment of rotation axis and reorientation of the bulge will continue (B3-B5) until the elastic stresses in the lithosphere balance the load-induced 
forcing. In the final state (B5), the load will not reach the equator. In this case, the rotation axis is said to be stable since the system retains memory of its original rotational state.

Willemann (1984) and Matsuyama et al. (2006) both treated the equilibrium form of the system (B5) and demonstrated that the net TPW was a strong function of both the magnitude of the load and its initial latitudinal position (see also Daradich et al., 2008). The Matsuyama et al. (2006) theory also revealed a sensitivity of the net TPW to lithospheric thickness. Chan et al. (2011) derived a linearized theory to model the time dependence in the pole position from (B1)-(B5) under the assumption of "small" perturbations in the pole position from its initial state. In contrast, Harada (2012), Creveling et al. (2012) and Chan et al. (2014) extended the non-linear theory of Ricard (1993) to include stabilization by the remnant bulge.

Finally, Figure 1C (the focus of this study) considers the physics associated with TPW on a planet with an initialy unstressed, viscoelastic (i.e., finite viscosity) lithosphere subject to a mass loading (C1-C2). The pole position is once again perturbed away from the load, but this motion is resisted by elastic stresses in the lithosphere. Initially, that is, over time scales shorter than the relaxation time, $\tau_{\text {lith }}$, of the lithosphere, the system will follow the trajectory of Figure 1B (TPW in $\mathrm{C} 2$ is the same as in B2). For a loading that persists over much longer time scale than $\tau_{\text {lith }}$, the elastic stresses in the lithosphere (and hence the resistance to polar motion) will relax, and the load will, as in Figure 1A5, ultimately reach the equator. Thus, this case bridges the physics of TPW for the scenarios with (Figure 1B) and without (Figure 1A) remnant bulge stabilization. Furthermore, while the equilibrium figure of the planet in Figure 1C is identical to that in Figure 1A, the presence of a high viscosity lithosphere will, as we demonstrate below, exert a dominant control on long-term, time-dependent TPW toward such an equilibrium state.

An issue of terminology arises. One might call the transient stabilization associated with the viscoelastic lithosphere in Figure $1 \mathrm{C}$ a remnant bulge, but the term was originally introduced by Willemann (1984) to denote a permanent stabilization in the case of a purely elastic lithosphere (Figure 1B). Alternatively, one might consider this stabilization as being one aspect of the transient rotational bulge. However, a high viscosity lithosphere will adjust to a new orientation of the rotation axis on a time scale that is much longer than the adjustment of the bulk mantle in response to TPW. Indeed, the theoretical derivation below makes explicit use of this large separation in time scales. Since the short time scale behaviour in Figure 1C is so distinct from the long time scale behavior, and since the time scale of the transition between these two states is governed by the viscosity of the lithosphere, which can 
(in theory) be arbitrarily high, we will generalize the Willemann (1984) terminology, and use the term 'transient remnant bulge' to describe the stabilization of rotation associated with incomplete relaxation of a high viscosity lithosphere. The term 'permanent remnant bulge' will, in contrast, be used when discussing stabilization of rotation by a purely elastic lithosphere.

In the next section we derive a rotational stability theory for TPW on a planet with a viscoelastic lithosphere. Our derivation provides a generalization of existing theory for timedependent TPW and, in this regard, the bridge described above will be highlighted by considering the special cases of a low viscosity ( $\left.\operatorname{short} \tau_{\text {lith }}\right)$ and infinite viscosity $\left(\tau_{\text {lith }}=\infty\right)$ lithosphere. In this section we have highlighted the physics of TPW on terrestrial planets by considering a load with magnitude and position relative to the solid Earth that remain fixed in time. We emphasize that the derivation below permits a loading with arbitrary time history, and in that case TPW will be controlled by the interplay between mantle and lithosphere relaxation times and the time scale(s) of the loading.

\section{Mathematical Theory}

Let us assume that in the unperturbed state, a planet with a (finite viscosity) viscoelastic lithosphere has a rotation vector $[0,0, \Omega]$ in a global geocentric Cartesian co-ordinate system, and that in the perturbed state the elements of the rotation vector are given by $\omega_{i}$, for $i=1,3$. We will also define the rotational driving potential as the perturbation in the centrifugal potential associated with TPW from this initial state. The total inertia tensor associated with the loaded system may be expressed as:

$I_{i j}(t)=I_{0} \delta_{i j}+\frac{k_{f}^{T, *} a^{5}}{3 G} \Omega^{2}\left(\delta_{i 3}-\frac{1}{3}\right) \delta_{i j}+\frac{k^{T}(t, V E) a^{5}}{3 G} *\left[\left(\omega_{i}(t) \omega_{j}(t)-\frac{1}{3} \omega^{2}(t) \delta_{i j}\right)-\Omega^{2}\left(\delta_{i 3}-\frac{1}{3}\right) \delta_{i j}\right]+I_{i j}^{L}(t)$

where $a$ is the mean radius of the planet and $G$ is the universal gravitational constant. In this equation, the first two terms on the right-hand-side (RHS) sum to give the inertia tensor of the initial, hydrostatic form of the planet (Figure 1C1) (the second of these terms represents the initial rotational bulge of the planet), the third term on the RHS, involving a time convolution (denoted by the asterisk), is the inertia tensor perturbation driven by the rotational driving potential, and the final term is the inertia tensor perturbation due to the direct effect of the mass redistribution (i.e., the load).

The third term is expressed as a time convolution of the perturbed centrifugal potential 
(the term in the square brackets) with the viscoelastic tidal (or tidal effective) $k$ Love number (Peltier, 1974). The latter is written as:

$$
k^{T}(t, V E)=k^{T, E} \delta(t)+\sum_{j=1}^{J} r_{j} e^{-s_{j} t}
$$

where the first term on the RHS is the elastic component of the Love number and the second is the non-elastic component, expressed as a sum of $J$ normal modes of pure exponential decay, with amplitude $r_{j}$ and inverse decay times $s_{j}$. We have used the symbol $V E$ in these expressions to remind the reader that the planetary model has a finite viscosity viscoelastic lithosphere, not a purely elastic lithosphere. In the Laplace transform domain, the Love number is given by

$$
k^{T}(s, V E)=k^{T, E}+\sum_{j=1}^{J} \frac{r_{j}}{s+s_{j}}
$$

The parameter $k_{f}^{T, *}$ in equation (1) is the so-called fluid Love number. An expression for this number can be derived by convolving the time dependent form of the $k$ Love number with a Heaviside loading and computing the infinite time response, or by setting $s=0$ in the $s$-domain form of the Love number. In either case, we obtain:

$$
k_{f}^{T, *}=k^{T, E}+\sum_{j=1}^{J} \frac{r_{j}}{s_{j}}
$$

Here, the asterisk is used to indicate that, for the planetary model we are considering, the fluid state of the planet has no elastic strength. The same would not be true for a planet with a purely elastic (infinite viscosity) lithosphere. In that case, the fluid Love number would be a function of the thickness of the elastic lithosphere.

While Harada (2012), Creveling et al. (2012) and Chan et al. (2014) derive the same governing equation for TPW in the presence of an elastic lithosphere, the extended derivation in this study closely parallels the derivation of Chan et al. (2014). In this regard, the main difference in the starting equation (1) used here versus that of Chan et al. (2014) is the appearance of the term $k^{T}(t, V E)$. The Chan et al. (2014) expression replaces this Love number with one that is computed from a planetary model with a purely elastic lithosphere, which they denoted as $k^{T}(t, L T)$, where $L T$ represents the thickness of this lithosphere. In that case, the third term on the RHS of equation (1) would guarantee that the system cannot re-establish the hydrostatic form of the initial state unless the pole returns to its original position. Thus, the Chan et al. (2014) expression, following earlier work by Willemann (1984) 
and Matsuyama et al. (2006), introduces a permanent remnant bulge that provides the rotating system with a memory of its initial rotational state. This remnant bulge arises through the difference between the long-term (fluid) limit of the Love number they adopted, $k^{T}(t, L T)$, and the parameter $k_{f}^{T, *}$ that appears in the second term on the RHS of equation (1).

The expression for the inertia tensor adopted by Ricard et al. (1993) differs from equation (1) by using $k^{T}(t, L T)$ in the third term on the RHS and adopting a fluid Love number in the second term on the RHS that includes an elastic lithosphere of thickness $L T$ (i.e., the asterisk we have used to indicate a fluid Love number computed for a model with no elastic lithosphere is dropped). In that case, there would be no remnant bulge stabilization since the system can readjust perfectly to its original form around the new pole position, but this original form is not hydrostatic. In this case, their expression and ours would be the same only in the case where the former assumes that $L T=0$. However, a more fundamental difference between our approach and theirs comes in the next step, where we seek an approximate expression for equation (3) in the case of small $s$.

We can rewrite equation (3) to isolate the normal mode(s) associated with the viscoelastic lithosphere:

$$
k^{T}(s, V E)=k^{T, E}+\sum_{j=1}^{J-1} \frac{r_{j}}{s+s_{j}}+\frac{r_{l i t h}}{s+s_{l i t h}}
$$

Under the assumption that we are considering processes with a time scale $\tau>1 / s_{j}$ for all modes other than this specific lithosphere mode (i.e., for $j=1, J-1$ ), we can approximate equation (5) as:

$$
k^{T}(s, V E) \approx k_{f}^{T, *}(1-s T)-\frac{r_{l i t h}}{s_{\text {lith }}} \frac{s}{\left(s+s_{\text {lith }}\right)}
$$

where

$$
T=\frac{1}{k_{f}^{T, *}} \sum_{j=1}^{J-1} \frac{r_{j}}{\left(s_{j}\right)^{2}}
$$

Ricard et al. (1993) did not consider a high viscosity, viscoelastic lithosphere, and their approximation to $k^{T}(s, V E)$ included only the first term on the RHS of equation (6), with $T$ defined in equation (7) as a sum over all modes.

The expression (6) assumes that the relaxation time of the lithosphere is significantly longer than other normal modes active in the system. Our expression for the Love number $k^{T}(s, V E)$ then involves the small $s$ (long time) limit of relaxation modes characterized by 
relatively short relaxation times (the first term on the RHS of equation 6), and the full contribution from the lithospheric mode. This decomposition can be trivially generalized to incorporate other normal modes that have very long relaxation times (whether these are associated with the lithosphere or not): the second term on the RHS of equation (6) would, in this more general case, be replaced with a summation over all such modes, and these modes would be excluded from the summation in equation (7). We return to this point below.

Next, we focus on the third term on the RHS of equation (1) involving a time convolution between the $k$ Love number and the rotational driving potential. We can proceed by transforming this term into the Laplace transform domain, applying the convolution theorem, adopting the approximation (6), and inverting back into the time domain. This sequence may be expressed as:

$$
\begin{aligned}
\frac{k^{T}(t, V E) a^{5}}{3 G} *[ & \left.\left(\omega_{i}(t) \omega_{j}(t)-\frac{1}{3} \omega^{2}(t) \delta_{i j}\right)-\Omega^{2}\left(\delta_{i 3}-\frac{1}{3}\right) \delta_{i j}\right] \\
= & \frac{a^{5}}{3 G} \mathcal{L}^{-1}\left(k^{T}(s, V E)\left[\left(\omega_{i}(s) \omega_{j}(s)-\frac{1}{3} \omega^{2}(s) \delta_{i j}\right)-\frac{1}{s} \Omega^{2}\left(\delta_{i 3}-\frac{1}{3}\right) \delta_{i j}\right]\right) \\
= & \frac{a^{5}}{3 G} \mathcal{L}^{-1}\left(\left[k_{f}^{T, *}(1-s T)-\frac{r_{l i t h}}{s_{l i t h}} \frac{s}{\left(s+s_{l i t h}\right)}\right]\left[\left(\omega_{i}(s) \omega_{j}(s)-\frac{1}{3} \omega^{2}(s) \delta_{i j}\right)-\frac{1}{s} \Omega^{2}\left(\delta_{i 3}-\frac{1}{3}\right) \delta_{i j}\right]\right) \\
= & \frac{k_{f}^{T, *} a^{5}}{3 G}\left[\left(\omega_{i}(t) \omega_{j}(t)-\frac{1}{3} \omega^{2}(t) \delta_{i j}\right)-\Omega^{2}\left(\delta_{i 3}-\frac{1}{3}\right) \delta_{i j}\right] \\
& -\frac{k_{f}^{T, *} a^{5}}{3 G} T\left[\dot{\omega}_{i}(t) \omega_{j}(t)+\omega_{i}(t) \dot{\omega}_{j}(t)-\frac{2}{3} \omega_{l} \dot{\omega}_{l} \delta_{l l}\right] \\
& \quad-\frac{a^{5}}{3 G} \frac{r_{l i t h}}{s_{l i t h}} \mathcal{L}^{-1}\left(\frac{s}{\left(s+s_{l i t h}\right)}\left[\left(\omega_{i}(s) \omega_{j}(s)-\frac{1}{3} \omega^{2}(s) \delta_{i j}\right)-\frac{1}{s} \Omega^{2}\left(\delta_{i 3}-\frac{1}{3}\right) \delta_{i j}\right]\right)
\end{aligned}
$$

where $\mathcal{L}$ represents the Laplace transform operator. By rewriting $s /\left(s+s_{\text {lith }}\right)$ as $1-s_{\text {lith }} /(s+$ $\left.s_{\text {lith }}\right)$, the inverse transform in the last term of equation (8) becomes:

$$
\begin{aligned}
\mathcal{L}^{-1}\left(\frac{s}{\left(s+s_{l i t h}\right)}\right. & {\left.\left[\left(\omega_{i}(s) \omega_{j}(s)-\frac{1}{3} \omega^{2}(s) \delta_{i j}\right)-\frac{1}{s} \Omega^{2}\left(\delta_{i 3}-\frac{1}{3}\right) \delta_{i j}\right]\right) } \\
& =\left[\delta(t)-s_{l i t h} e^{-s_{l i t h} t}\right] *\left[\left(\omega_{i}(t) \omega_{j}(t)-\frac{1}{3} \omega^{2}(t) \delta_{i j}\right)-\Omega^{2}\left(\delta_{i 3}-\frac{1}{3}\right) \delta_{i j}\right]
\end{aligned}
$$

With no loss of generality, we can express the time dependence of the perturbed centrifugal potential as

$$
\Delta \Phi_{i j}(t)=\sum_{l=0}^{L} \delta \Phi_{i j}^{l} H\left(t-t_{l}\right)=\left(\omega_{i}(t) \omega_{j}(t)-\frac{1}{3} \omega^{2}(t) \delta_{i j}\right)-\Omega^{2}\left(\delta_{i 3}-\frac{1}{3}\right) \delta_{i j}
$$


where $H$ is the Heaviside step function. Using this expression, the time convolution in equation (9) can be performed analytically, yielding

$\mathcal{L}^{-1}\left(\frac{s}{\left(s+s_{l i t h}\right)}\left[\left(\omega_{i}(s) \omega_{j}(s)-\frac{1}{3} \omega^{2}(s) \delta_{i j}\right)-\frac{1}{s} \Omega^{2}\left(\delta_{i 3}-\frac{1}{3}\right) \delta_{i j}\right]\right)=\sum_{l=0}^{L} \delta \Phi_{i j}^{l} e^{-s_{l i t h}\left(t-t_{l}\right)} H\left(t-t_{l}\right)$

Combining equations (1), (8) and (11) yields our final expression for the total inertia tensor:

$$
\begin{aligned}
I_{i j}(t)=I_{0} \delta_{i j}+\frac{k_{f}^{T, *} a^{5}}{3 G}\left(\omega_{i}(t) \omega_{j}(t)\right. & \left.-\frac{1}{3} \omega^{2}(t) \delta_{i j}\right)-\frac{k_{f}^{T, *} a^{5}}{3 G} T\left[\dot{\omega}_{i}(t) \omega_{j}(t)+\omega_{i}(t) \dot{\omega}_{j}(t)-\frac{2}{3} \omega_{l} \dot{\omega}_{l} \delta_{l l}\right] \\
& -\frac{a^{5}}{3 G} \frac{r_{l i t h}}{s_{l i t h}} \sum_{l=0}^{L} \delta \Phi_{i j}^{l} e^{-s_{l i t h}\left(t-t_{l}\right)} H\left(t-t_{l}\right)+I_{i j}^{L}(t)
\end{aligned}
$$

We note that Daradich (2007) derived an expression similar to equation (12), with the exception that the term associated with the delayed viscous adjustment of the rotational bulge was not included.

As we noted below equation (7) we can generalize the above expression in the case of multiple modes with very long relaxaton times. For the case of $\mathrm{N}$ such modes, equation (12) would be written as:

$$
\begin{aligned}
I_{i j}(t)=I_{0} \delta_{i j}+\frac{k_{f}^{T, *} a^{5}}{3 G}\left(\omega_{i}(t) \omega_{j}(t)\right. & \left.-\frac{1}{3} \omega^{2}(t) \delta_{i j}\right)-\frac{k_{f}^{T, *} a^{5}}{3 G} T_{N}\left[\dot{\omega}_{i}(t) \omega_{j}(t)+\omega_{i}(t) \dot{\omega}_{j}(t)-\frac{2}{3} \omega_{l} \dot{\omega}_{l} \delta_{l l}\right] \\
& -\frac{a^{5}}{3 G} \sum_{n=0}^{N-1} \frac{r_{J-n}}{s_{J-n}} \sum_{l=0}^{L} \delta \Phi_{i j}^{l} e^{-s_{J-n}\left(t-t_{l}\right)} H\left(t-t_{l}\right)+I_{i j}^{L}(t)
\end{aligned}
$$

where

$$
T_{N}=\frac{1}{k_{f}^{T, *}} \sum_{j=1}^{J-N} \frac{r_{j}}{\left(s_{j}\right)^{2}}
$$

With these equations for the inertia tensor in hand, the fundamental underlying assumption we make to solve for the time-varying rotation axis is that the time scale being considered is long enough that the rotation axis stays aligned with the principle axis of inertia (Ricard et al., 1993; Harada, 2012; Chan et al., 2014). In this case, our calculations of TPW are based on diagonalizing equation (12) (or equation 13) and computing the time-varying orientation of the principle axis of inertia. 


\subsection{Limiting Cases}

We next consider two limiting cases of equation (12). The first involves a scenario with no lithosphere and the second treats the case with an elastic lithosphere.

\section{No lithosphere}

If the lithosphere has no viscosity contrast with the underlying mantle, the lithospheric mode isolated in the above expression would disappear. In this case, equation (12) becomes:

$$
\begin{aligned}
I_{i j}(t)=I_{0} \delta_{i j}+\frac{k_{f}^{T, *} a^{5}}{3 G} & \left(\omega_{i}(t) \omega_{j}(t)-\frac{1}{3} \omega^{2}(t) \delta_{i j}\right) \\
& \quad-\frac{k_{f}^{T, *} a^{5}}{3 G} T\left[\dot{\omega}_{i}(t) \omega_{j}(t)+\omega_{i}(t) \dot{\omega}_{j}(t)-\frac{2}{3} \omega_{l} \dot{\omega}_{l} \delta_{l l}\right]+I_{i j}^{L}(t)
\end{aligned}
$$

and the summation in equation (7) would now include all modes of relaxation. This expression matches equation (10) of Ricard et al. (1993) for the case of no lithosphere. To derive their result in the case of an elastic lithosphere, one simply replaces the two appearances of $k_{f}^{T, *}$ in the above equation with the fluid tidal Love number computed for a model with such a lithosphere, which we may write as $k_{f}^{T}(L T)$. We emphasize that even in the latter case there is no permanent remnant bulge stabilization since the system does not retain a memory of the original rotational state.

\section{An elastic lithosphere}

For the case of a planet with an elastic (infinite viscosity) lithosphere, we take the limit $s_{l i t h} \rightarrow 0$ in equation (12). This limit yields, using equation (10):

$$
\begin{aligned}
I_{i j}(t)=I_{0} \delta_{i j}+\frac{a^{5}}{3 G}\left(k_{f}^{T, *}-\frac{r_{l i t h}}{s_{l i t h}}\right) & \left(\omega_{i}(t) \omega_{j}(t)-\frac{1}{3} \omega^{2}(t) \delta_{i j}\right)-\frac{a^{5}}{3 G} \frac{r_{l i t h}}{s_{l i t h}} \Omega^{2}\left(\delta_{i 3}-\frac{1}{3}\right) \delta_{i j} \\
& -\frac{k_{f}^{T, *} a^{5}}{3 G} T\left[\dot{\omega}_{i}(t) \omega_{j}(t)+\omega_{i}(t) \dot{\omega}_{j}(t)-\frac{2}{3} \omega_{l} \dot{\omega}_{l} \delta_{l l}\right]+I_{i j}^{L}(t)
\end{aligned}
$$

We can compare this expression, which involves a permanent remnant bulge stabilization, with equation (16) of Chan et al. (2014):

$$
\begin{array}{r}
I_{i j}(t)=I_{0} \delta_{i j}+\frac{a^{5}}{3 G} k_{f}^{T}(L T) \\
\left(\omega_{i}(t) \omega_{j}(t)-\frac{1}{3} \omega^{2}(t) \delta_{i j}\right)-\frac{a^{5}}{3 G}\left(k_{f}^{T}(L T)-k_{f}^{T, *}\right) \Omega^{2}\left(\delta_{i 3}-\frac{1}{3}\right) \delta_{i j} \\
-\frac{k_{f}^{T}(L T) a^{5}}{3 G} T_{c}\left[\dot{\omega}_{i}(t) \omega_{j}(t)+\omega_{i}(t) \dot{\omega}_{j}(t)-\frac{2}{3} \omega_{l} \dot{\omega}_{l} \delta_{l l}\right]+I_{i j}^{L}(t)
\end{array}
$$


We note that the parameter $T$ in equation (16) includes the term $k_{f}^{T, *}$ in its denominator (see equation 7) and this cancels with the $k_{f}^{T, *}$ that it is multiplied by. A similar cancellation occurs in the product $k_{f}^{T}(L T) T_{c}$ (Chan et al., 2014). Thus the fourth term on the RHS of both equations are identical. ( $T_{c}$ is analogous to the parameter in equation (7) with the exception that $k_{f}^{T, *}$ is replaced by $k_{f}^{T}(L T)$ and the sum includes all normal modes in the elastic lithosphere case.)

The remaining differences between the two expressions involve the second and third terms on the RHS of each. A comparison indicates that equations (16) and (17) are the same if:

$$
k_{f}^{T, *}-k_{f}^{T}(L T)=\frac{r_{l i t h}}{s_{l i t h}}
$$

That is, the two equations are identical only if the difference between the $k$ tidal fluid Love numbers in the case of a planetary model with a (finite viscosity) viscoelastic lithosphere and a model with an (infinite viscosity) elastic lithosphere is equal to the ratio of the modal amplitude and inverse decay time of the lithospheric relaxation mode associated with the first of these models. We numerically verify this heretofore unrecognized relationship in the next section.

\subsection{Prescribing the Loading Term, $I_{i j}^{L}(t)$}

In the above derivation, the loading term has been left in the most general, time-dependent form $I_{i j}^{L}(t)$. TPW can be driven by either an internal or external loading, or some combination of the two. In the case of an internal load, the time history of the load inertia tensor might be calculated, for example, using a simulation of convective flow in the planetary interior. For an external loading, the load inertia tensor may be written as a convolution involving the inertia tensor perturbation due to the surface mass load on a rigid planet, $I_{i j}^{R}(t)$, and the viscoelastic $k$ load Love number at spherical harmonic degree two (Chan et al., 2014):

$$
I_{i j}^{L}(t)=I_{i j}^{R}(t) *\left[\delta(t)+k^{L}(t, V E)\right]
$$

where the second term in square brackets yields the inertia perturbation due to planetary deformation driven by the surface mass loading. In analogy with equation (2), the $k$ load Love number can be written as:

$$
k^{L}(t, V E)=k^{L, E} \delta(t)+\sum_{j=1}^{J} r_{j}^{\prime} e^{-s_{j} t}
$$


The Love numbers $k^{L}$ and $k^{T}$ share the set of inverse decay times, $s_{j}$, but have distinct modal amplitudes $r_{j}^{\prime}$ and $r_{j}$, respectively.

In Section 2 we discussed the physics of TPW using the simple example of a load emplaced and retained for all time. This special case raises an interesting issue when considering a surface mass loading. Specifically, if a surface mass load is applied on a planet with a viscoelastic lithosphere, and remains fixed for all time, then the inertia tensor perturbation due to the loading, $I_{i j}^{L}(t)$, will approach zero as the mass load approaches a state of complete isostatic compensation (i.e., the fluid $k$ load number is -1 in the case of a finite viscosity lithosphere). In such a scenario, it is possible that the load driving TPW might be fully compensated before it reaches the position shown in Figure 1C4 (i.e., TPW may cease in this case before the load reaches the equator).

\section{Results and Discussion}

We will illustrate the results of our mathematical treatment using Mars as an example. For this purpose, we adopt the elastic and density structure of the planet derived by Zharkov and Gudkova (2005). The viscosity profile will be discretized into two isoviscous layers: a lithosphere of prescribed thickness and viscosity, and a sub-lithospheric region with a viscosity set to $10^{21} \mathrm{~Pa}$ s. The latter is likely an upper bound on the viscosity of the Martian mantle (Hauck and Phillips, 2002; Williams and Nimmo, 2004; Breuer and Spohn, 2006). The results discussed below would not be altered if we chose to use a lower value for the mantle viscosity since viscous relaxation in that region is fast enough in the case of a viscosity of $10^{21} \mathrm{~Pa} \mathrm{~s}$ that it provides no stabilization on the loading time scales treated below (see Chan et al., 2014).

Figure 2 shows the inverse decay times, that is, the parameters $s_{j}$ in equation (2), for four different models of Mars that are distinguished on the basis of the adopted viscosity of a lithosphere of thickness $110 \mathrm{~km}$ (as labeled on the x-axis). Specifically, we consider lithospheric viscosities of $10^{25} \mathrm{~Pa} \mathrm{~s}, 10^{26} \mathrm{~Pa} \mathrm{~s}, 10^{27} \mathrm{~Pa} \mathrm{~s}$ and inifinity (i.e., a purely elastic lithosphere). The dotted lines join specific mode families, each of which is associated with a different aspect of the internal structure of Mars. As an example, the top line on the figure is the so-called M0 mode, the fundamental relaxation mode associated with the density contrast at the surface of the planet. This mode carries $85 \%$ of the total strength of the non-elastic component of the fluid Love number (equation 4). A comparison of modes computed with finite and infinite viscosity lithospheres indicates that the cluster of points encircled by the ellipses represent modes that arise from the adoption of a viscoelastic lithosphere. These modes have decay 


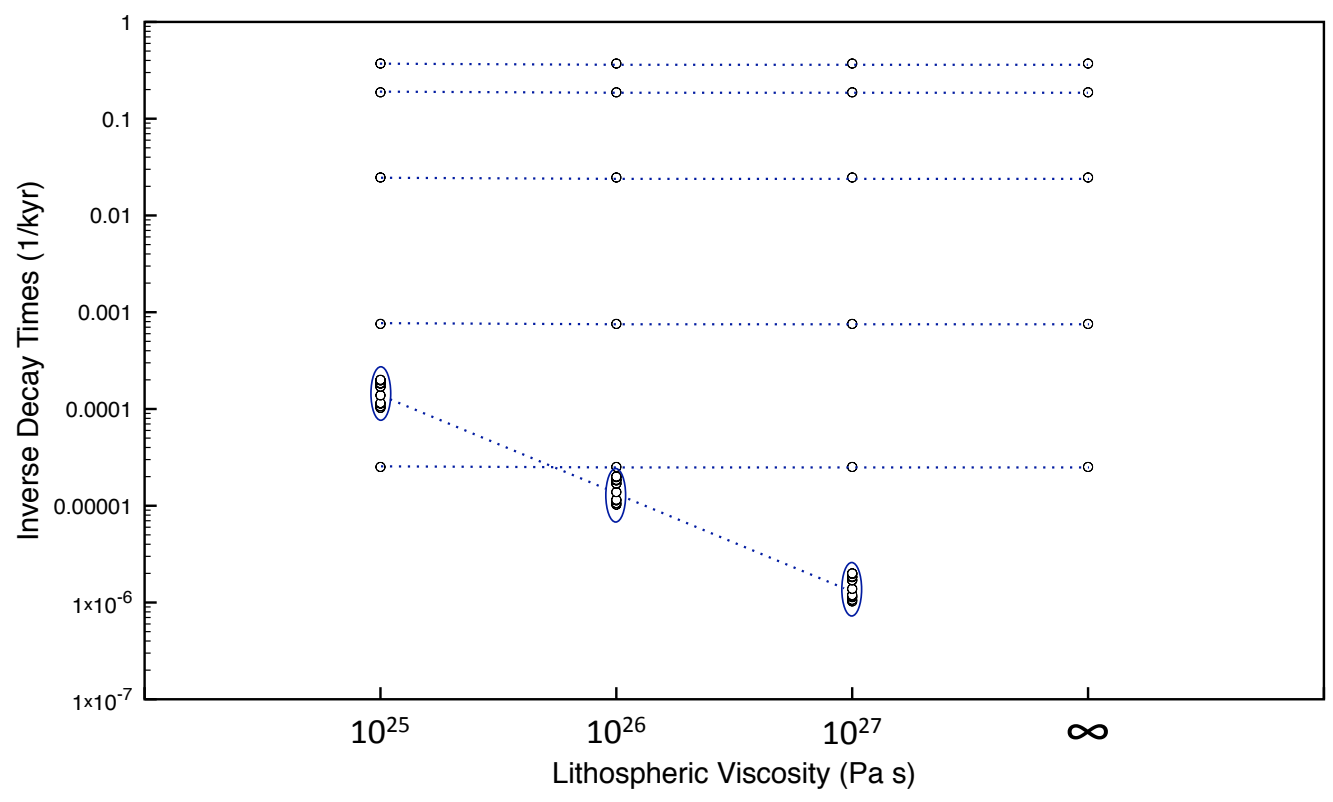

Figure 2. Inverse decay times, $s_{j}$ (equation 2), for four different models of Mars distinguished on the basis of the viscosity of the lithosphere, as given by the abscissa. All models have a lithospheric thickness of $110 \mathrm{~km}$, and density and elastic structure taken from Zharkov and Gudkova (2005). The dotted lines join mode families associated with a specific feature of the Mars model (see text).

times that reflect the relaxation (or Maxwell) time of the lithosphere: $\sim 10^{7} \mathrm{yr}$ for lithospheric viscosity of $10^{25} \mathrm{~Pa} \mathrm{~s} ; \sim 10^{8} \mathrm{yr}$ for $10^{26} \mathrm{~Pa} \mathrm{~s}$; and $\sim 10^{9} \mathrm{yr}$ for $10^{27} \mathrm{~Pa} \mathrm{~s}$. These modes are the $N$ modes to be included in calculating the last term on the RHS of equation (13).

We can use these inverse decay times, together with their associated mode amplitude $r_{j}$, to test the validity of the approximation in equation (18) (where $r_{l i t h} / s_{\text {lith }}$ is replaced by a sum that includes all the modes of very long decay times, i.e., the mode clusters in Figure 2) which we inferred on the basis of equating equations (16) and (17). The results of a comparison between $k_{f}^{T, *}-k_{f}^{T}(L T)$ and the sum of terms $r_{j} / s_{j}$ for all modes associated with lithospheric relaxation are summarized in Table 1 . The first three rows of the table are the Mars models with viscoelastic lithospheres treated in Figure 2. These results are augmented with three additional models with a lithospheric viscosity of $10^{26} \mathrm{~Pa} \mathrm{~s}$ and thicknesses of $48 \mathrm{~km}, 164$ $\mathrm{km}$, and $270 \mathrm{~km}$. It is clear from Table 1 that equation (18) is very accurate, and thus that the $s_{l i t h} \rightarrow 0$ (high lithospheric viscosity) limit of our rotational stability theory converges, 
as it should, to the theory of Chan et al. (2014) (see also Harada (2012) and Creveling et al. (2012)) for TPW on planetary models with an elastic lithosphere.

Table 1

\begin{tabular}{lllll}
\hline $\begin{array}{l}\text { Lithospheric } \\
\text { viscosity (Pa s) }\end{array}$ & $\begin{array}{l}\text { Lithospheric } \\
\text { thickness }(\mathrm{km})\end{array}$ & $k_{f}^{T, *}-k_{f}^{T}(L T)$ & $\begin{array}{l}\text { Sum of } \\
r_{\text {lith }} / s_{\text {lith }}\end{array}$ & $\begin{array}{l}\text { Percent } \\
\text { error }\end{array}$ \\
\hline $10^{25}$ & 110 & 0.1834 & 0.1840 & 0.327 \\
\hline $10^{27}$ & 110 & 0.1834 & 0.1833 & -0.0545 \\
\hline $10^{26}$ & 110 & 0.1834 & 0.1835 & 0.0545 \\
\hline $10^{26}$ & 48 & 0.0792 & 0.0792 & 0.0126 \\
\hline $10^{26}$ & 164 & 0.2606 & 0.2605 & -0.0384 \\
\hline $10^{26}$ & 270 & 0.3909 & 0.3908 & -0.0256 \\
\hline
\end{tabular}

We note that the approximation in equation (18) breaks down as the lithospheric viscosity of the Mars model is reduced below $10^{25} \mathrm{~Pa}$ s to values that yielded inverse decay times close to those of other modes of non-negligible power. However, reducing the lithospheric viscosity to this extent violates the underlying assumption of our derivation in equation (6), that the timescale of relaxation of the lithospheric modes is much longer than that of the bulk mantle.

We turn next to prescribing the load $I_{i, j}^{L}(t)$ in equation (13). We use a load with an amplitude following a hyperbolic tangent time history, with a ramp up taking 10 Myr. If we write $I_{i, j}^{L}(t)=I_{i, j}^{L} \mathcal{F}(t)$, then:

$$
\mathcal{F}(t)=0.5 \tanh \left(\frac{6 \mathrm{t}-30}{10}\right)+0.5
$$

where $t$ is time in units of Myr since the onset of loading. We will drive TPW on Mars using a load initially oriented $45^{\circ}$ from the equator. (Since we are specifying the time varying inertia tensor perturbation, rather than computing it from a convection simulation or through a surface loading calculation as in equation (20), one may interpret the load as an effective forcing that can be of either internal or external origin, or a combination of both.) In the limit of very long time, this emplaced load will reach the equator and thus the total TPW will be $45^{\circ}$. We will present results below for viscoelastic lithospheres characterized by a range of thicknesses. For each thickness, we adopt a value for the load amplitude, $I_{i, j}^{L}$, such that if the 
lithosphere were elastic (i.e., if a permanent remnant bulge was active and equation 17 was being solved), the final TPW would be $22.5^{\circ}$.

Figure 3 shows predictions of TPW in response to the above loading based on various non-linear rotational stability theories. The light grey line was computed using the theory of Ricard et al. (1993) (equation 15, and Figure 1A), which includes stabilization by the delayed adjustment of the rotational bulge (third term on the RHS of equation 15), but no remnant bulge stabilization (transient or permanent). In this case, polar wander reorients the load to the equator in a few million years. The remaining lines on the figure were computed using a Mars model characterized by a lithospheric thickness of $110 \mathrm{~km}$. The dark grey line is a prediction based on the theory of Chan et al. (2014) (equation 17, Figure 1B), which includes a permanent remnant bulge stabilization and a transient stabilization associated with the adjustment of the rotational bulge (the third and fourth terms on the RHS of equation 17, respectively). As noted by Chan et al. (2014), time dependent TPW in this case closely tracks the equilibrium theory; that is, mantle viscosity below the lithosphere $\left(10^{21} \mathrm{~Pa} \mathrm{~s}\right)$ is sufficiently low that stabilization by the transient rotational bulge has little impact on the predicted TPW and the time dependence is largely governed by the 10 Myr ramp up in the mass load. The final TPW of $22.5^{\circ}$ is governed by a balance between the load-induced forcing and stabilization by the permanent remnant bulge.

The blue lines on Figure 3 are predictions based on the theory presented in this study (equation 13, Figure 1C). In all three cases, the TPW predictions initially follow the calculation based on Chan et al. (2014), but eventually the stabilization by a transient remnant bulge weakens and the pole position departs from this trend and moves toward the prediction based on the Ricard et al. (1993) theory. As one would expect, the time scale of this transition increases as the adopted viscosity of the lithosphere increases (see also Figure 2). For example, in the case of a lithosphere with viscosity $10^{25} \mathrm{~Pa} \mathrm{~s}$, characterized by a relaxation time scale of $\sim 10 \mathrm{Myr}$, the predicted TPW reaches $45^{\circ}$ in $\sim 50$ Myr. We have verified that the remaining two predictions, based on lithospheric viscosities of $10^{26} \mathrm{~Pa} \mathrm{~s}$ and $10^{27} \mathrm{~Pa}$ s, require a factor of $\sim 10$ and $\sim 100$ longer, respectively, to reach this state. Indeed, in the 300 Myr time span plotted on the figure, the latter simulation moves only $\sim 8^{\circ}$ off the prediction associated with a permanent remnant bulge stabilization. Chan et al. (2014) concluded that long-term TPW on Mars would have a time scale that reflected the time variability of the driving load, and, as we discussed in the context of the dark grey line in Figure 2, TPW could be accurately computed using an equilibrium rotational stability theory. That is, TPW would cease soon after the load ceased to change. Our predictions indicate, in contrast, that in the case of a 


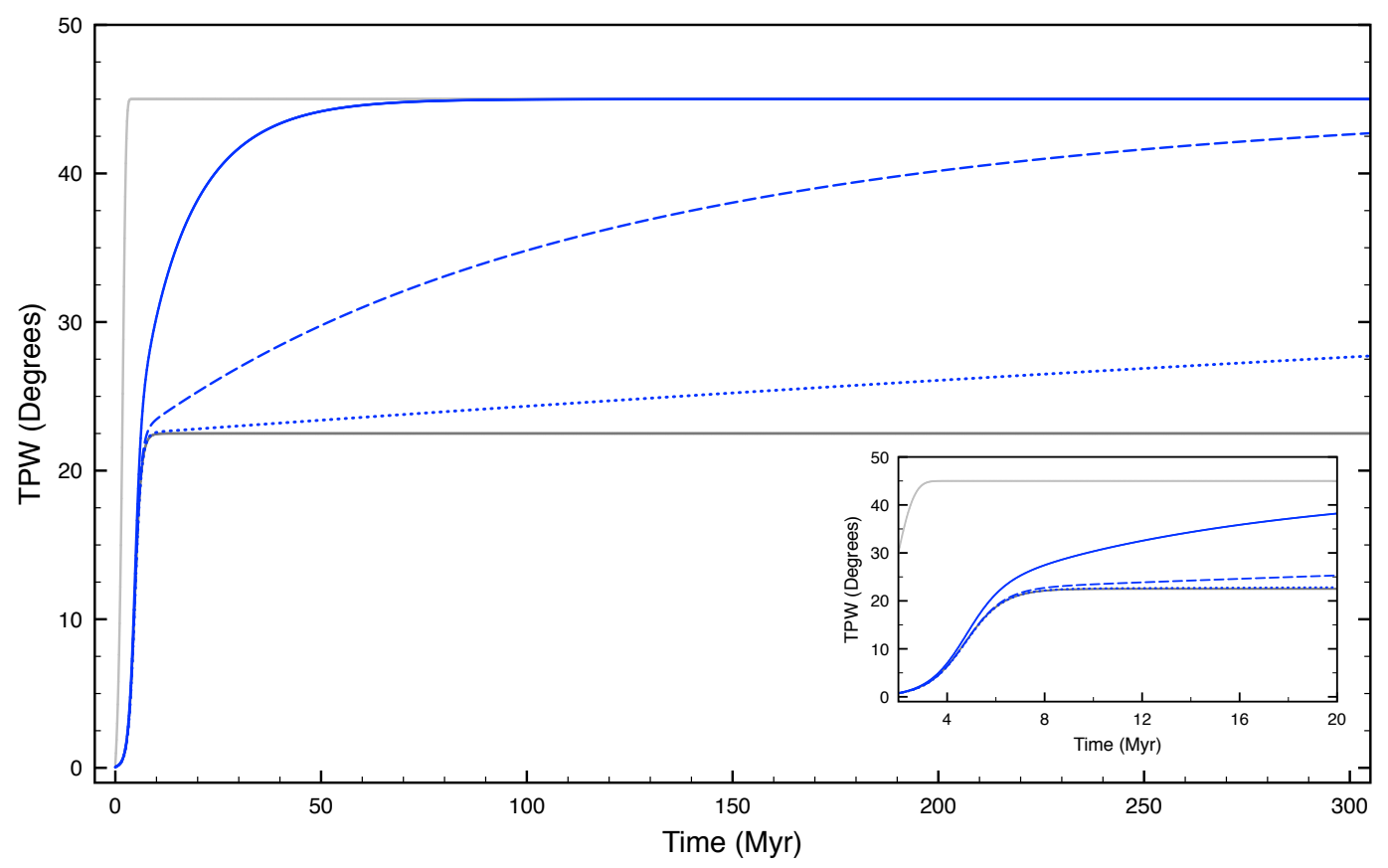

Figure 3. A comparison of results for various predictions of TPW in response to a mass load initially at a colatitude of $45^{\circ}$, and increasing from zero to a maximum amplitude over $10 \mathrm{Myr}$ (see text). Light grey line - prediction based on the theory of Ricard et al. (1993) (equation 15) that does not include stabilization by a remanent bulge. Dark grey line - prediction based on the theory of Chan et al. (2014) (equation 17), which includes permanent remnant bulge stabilization. Blue lines - predictions based on the stability theory derived in the present study (equation 13), which includes a transient remnant bulge stabilization, for lithospheric viscosities of $10^{25} \mathrm{~Pa} \mathrm{~s}$ (solid), $10^{26} \mathrm{~Pa} \mathrm{~s}$ (dashed), and $10^{27} \mathrm{~Pa} \mathrm{~s}$ (dotted). The inset shows a detail of the first $20 \mathrm{Myr}$ of the simulations. All calculations assume a lithospheric thickness of $110 \mathrm{~km}$, with the exception of the light grey line which was computed using a model with a uniform mantle viscosity and no elastic lithosphere.

lithosphere of viscosity $10^{25}-10^{27} \mathrm{~Pa} \mathrm{~s}$, TPW on Mars may persist for time scales of order $10^{8}-10^{10}$ yr after the emplacement of a load.

These results raise the question of whether the thickness of the adopted lithosphere has any impact on the time scale of the predicted TPW. To explore this issue, we repeated the prediction in Figure 3 based on a lithospheric viscosity of $10^{26} \mathrm{~Pa} \mathrm{~s}$ (dashed blue line) for three alternate plate thicknesses: $48 \mathrm{~km}, 164 \mathrm{~km}$, and $270 \mathrm{~km}$ (these cases were included in Table 1) and the results are shown in Figure 4. It is clear that the thickness of the viscoelastic 


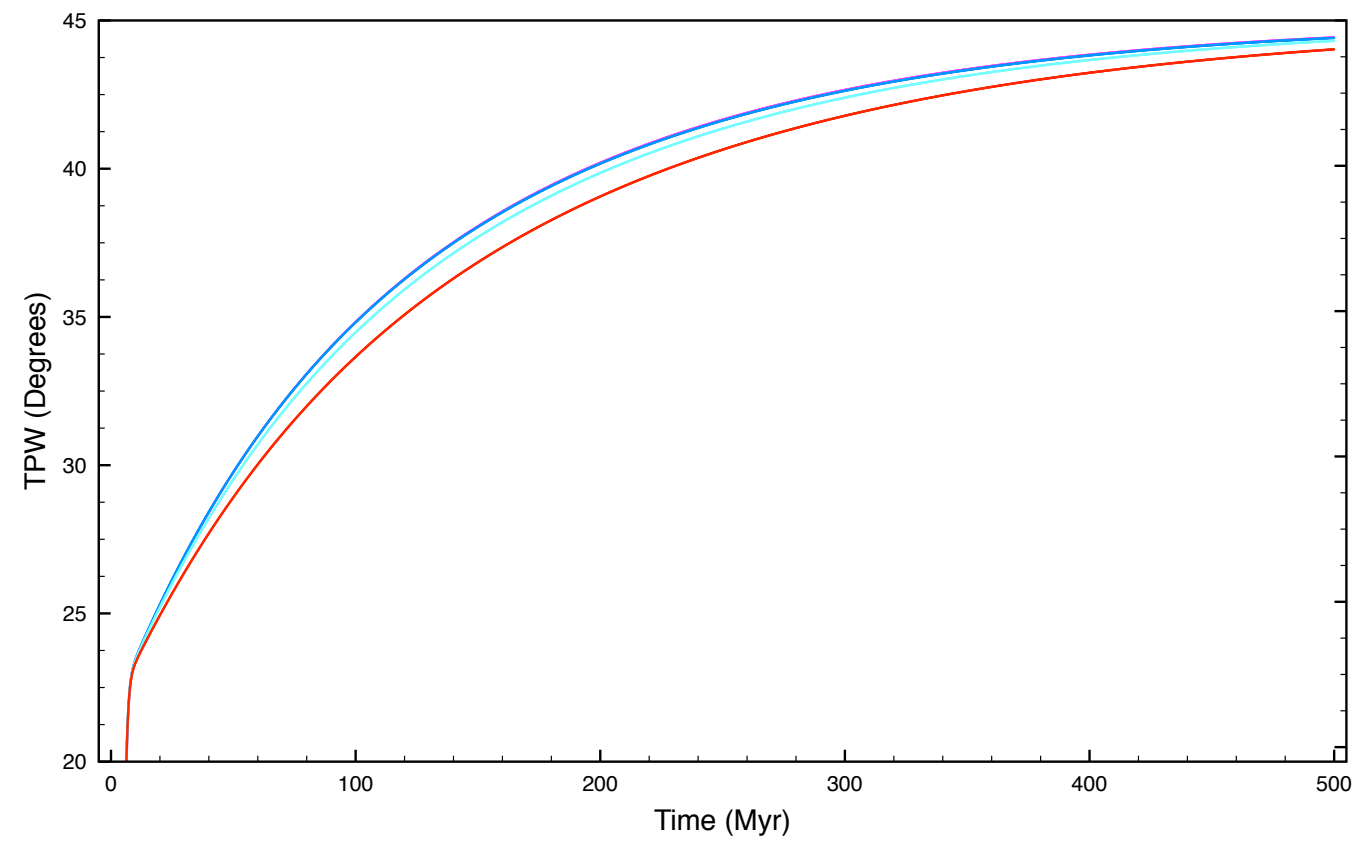

Figure 4. A comparison of results for various predictions of TPW in response to a mass load initially at a colatitude of $45^{\circ}$, and increasing from zero to a maximum amplitude over $10 \mathrm{Myr}$ (see text). All calculations are based on the rotational stability theory derived in the present study (equation 13) and a lithospheric viscosity of $10^{26} \mathrm{~Pa} \mathrm{~s}$. The lines are distinguished on the basis of the thickness of the lithosphere: Blue $(110 \mathrm{~km})$, cyan $(164 \mathrm{~km})$, and red $(270 \mathrm{~km})$. A fourth line, magenta $(48 \mathrm{~km})$ is not visible below the $110 \mathrm{~km}$ lithosphere results.

lithosphere exerts little control on the time scale of the predicted TPW relative to the much larger effect of changing the lithospheric viscosity.

\section{Conclusions}

We have described a non-linear rotational stability theory that considers TPW on terrestrial planets with viscoelastic lithospheres. The theory bridges treatments described in the canonical work of Ricard et al. (1993), and the more recent studies of Harada (2012), Creveling et al. (2012) and Chan et al. (2014), by incorporating a transient remnant bulge that acts to stabilize the rotation axis over a time scale connected to the relaxation time of the finite viscosity lithosphere.

We have illustrated the new theory using a simplified mass loading of Mars. A wide range of estimates exists for the thickness of the early and modern Martian lithosphere, and these estimates show regional variability (Sohl and Spohn, 1997; Plaut et al., 2007; Philips et al., 2008). More importantly, from the perspective of the present study, is the significant 
gravity anomaly associated with Tharsis (Zuber and Smith, 1997; Zuber et al., 2000), which suggests the lithosphere has not fully relaxed over the 3-4 billion years since the development of that feature (though one can not rule out that the isostatic disequilibrium reflects a more recent phase of volcanic loading). Similarly, while most of the topography on Mars' southern hemisphere appears to be in isostatic equilibrium, several large Noachian craters like Hellas are characterized by small gravity anomalies (Smith et al., 1999). Taken together, this evidence implies a lithospheric viscosity of at least $\sim 10^{27} \mathrm{~Pa}$ s, indicating that load emplacement and viscous relaxation of the lithosphere may drive TPW over the same, very long time scale.

We note that while our numerical examples have treated a lithosphere of constant viscosity, the rotational stability theory summarized by equation (13) may be applied to model TPW in the case of a lithosphere with a layered viscosity structure. However, Love number theory is not valid for a lithosphere with lateral variations in structure, including the plate boundaries that exist on Earth. Nevertheless, in such cases, significant insight may be gained by applying our theory and interpreting the (input) uniform thickness of the lithosphere as an effective thickness. Calculations in Creveling et al. (2012) suggest, for example, that breaks in the Earth's lithosphere (i.e., plate boundaries) reduce the effective lithospheric thickness in response to centrifugal forcing by TPW by an order of magnitude. The results in Figure 4 suggest that even with such a reduction, a broken, high viscosity lithosphere may still exert an important control on long-term TPW.

\section{ACKNOWLEDGMENTS}

We thank an anonymous reviewer and the Editor for their comments in regard to an earlier version of this manuscript. This work is supported in part by the M. Hildred Blewett Fellowship of the American Physical Society, www.aps.org (AD); the United States Department of Defense through the NDSEG Program (KM); the Natural Sciences and Engineering Research Council of Canada (N-HC); and Harvard University.

\section{REFERENCES}

Breuer, D., \& Spohn, T., 2006. Viscosity of the Martian mantle and its initial temperature: Constraints from crust formation history and the evolution of the magnetic field, Planet. Space Sci., 54, 153-169. Chan, N.-H., Mitrovica, J.X., Matsuyama, I., Creveling, J.R. \& Stanley, S., 2011. The rotational stability of a convecting Earth: Assessing inferences of rapid TPW in the late Cretaceous, Geophysics. J. Int., 187, 1319-1333. 
Chan, N.-H., Mitrovica, J.X., Daradich, A., Creveling, J.R., Matsuyama, I. \& Stanley, S., 2014. Timedependent rotational stability of dynamic planets with elastic lithospheres, J. Geophy. Res., 119, $169-188$.

Creveling, J.R., Mitrovica, J.X., Chan, N.-H., Latychev, K. \& Matsuyama, I., 2012. Mechanisms for oscillatory true polar wander, Nature, 491, 244-248.

Daradich, A.L., 2007. Dynamic topography of continents and rotational stability of planets with lithospheres, Ph.D. Thesis, University of Toronto, Toronto, Canada.

Daradich, A., Mitrovica, J.X., Matsuyama, I. \& Perron, J.T., 2008. Equilibrium rotational stability and figure of Mars, Icarus, 194, 463-475.

Gold, T., 1955. Instability of Earth's axis of rotation, Nature, 175, 526-529.

Goldreich, P. \& Toomre, A., 1969. Some remarks on polar wandering, J. Geophys. Res, 74, 2555-2567.

Harada, Y., 2012. Long-term polar motion on a quasi-fluid planetary body with an elastic lithosphere: Semi-analytic solutions of the time-dependent equation, Icarus, 220, 449-465.

Hauck, S.A., \& Phillips, R.J., 2002. Thermal and crustal evolution of Mars, J. Geophys. Res., 107, E7-5012.

Matsuyama, I., Mitrovica, J.X., Manga, M., Perron, J.T. \& Richards, M.A., 2006. Rotational stability of dynamic planets with elastic lithospheres, J. Geophys. Res., 111, E02003.

Mitrovica, J.X., Wahr, J.M., Matsuyama, I. \& Paulson, A., 2005. The rotational stability of an ice-age Earth, Geophys. J. Int., 161, 491-506.

Peltier, W.R., 1974. The impulse response of a Maxwell Earth, Rev. Geophys. Space Phys., 12, 649669.

Phillips, R.J., et al., 2008. Mars north polar deposits: Stratigraphy, age, and geodynamical response, Science, 320, 1182-1185.

Plaut, J.J., et al., 2007. Subsurface radar sounding of the south polar layered deposits of Mars, Science, 316, 92-95.

Ricard, Y., Spada, G., and Sabadini, R., 1993. Polar wander of a dynamic Earth, Geophys. J. Int., 113, 284-298.

Sabadini, R. \& Peltier, W.R., 1981. Pleistocene deglaciation and the Earth's rotation: implications for mantle viscosity, Geophys. J. Roy. Astron. Soc., 66, 553-578.

Sabadini, R., Yuen, D.A. \& Boschi, E., 1982. Interaction of cryospheric forcings with rotational dynamics has consequences for ice ages, Nature, 296, 338-341.

Smith, D.E., et al., 1999. The global topography of Mars and implications for surface evolution, Science, 284, 1495-1503.

Sohl, F., \& Spohn, T., 1997. The interior structure of Mars: Implications from SNC meteorites, J. Geophys. Res., 102, 1613-1635.

Tsai, V.C. \& Stevenson, D.J., 2007. Theoretical constraints on true polar wander, J. Geophys. Res, 112, B05415. 
Vermeersen, L.L.A. \& Sabadini, R., 1999. Polar wander, sea-level variations and ice age cycles, Surv. Geophys., 20, 415-440.

Willemann, R.J., 1984. Reorientation of planets with elastic lithospheres, Icarus, 60, 701-709.

Williams, J.-P., \& Nimmo, F., 2004. Thermal evolution of the Martian core: Implications for an early dynamo, Geology, 32(2), 97-100.

Wu, P. \& Peltier, W. R., 1984. Pleistocene deglaciation and the Earth's rotation: a new analysis, Geophys. J. Roy. Astron. Soc., 76, 753-791.

Zharkov, V. \& Gudkova, T., 2005. Construction of Martian interior model, Sol. Syst. Res., 39, 343373.

Zuber, M.T., \& Smith, D.E., 1997. Mars without Tharsis, J. Geophys. Res., 102, 28673-28685.

Zuber, M.T., et al., 2000. Internal structure and early thermal evolution of Mars from Mars Global Surveyor Topography and Gravity, Science, 287, 1788-1793. 